Board of Governors of the Federal Reserve System

International Finance Discussion Papers

Number 752

December 2002

Uncovered Interest Parity: It Works, But Not For Long

Alain P. Chaboud and Jonathan H. Wright

NOTE: International Finance Discussion Papers are preliminary materials circulated to stimulate discussion and critical comment. References in publications to International Finance Discussion Papers (other than an acknowledgment that the writer has had access to unpublished material) should be cleared with the author or authors. Recent IFDPs are available on the Web at www.federalreserve.gov/pubs/ifdp/. 


\title{
Uncovered Interest Parity: It Works, But Not For Long
}

Alain P. Chaboud and Jonathan H. Wright*

\begin{abstract}
The failure of uncovered interest parity can be ascribed to the existence of a risk premium. The size of this risk premium may shrink to zero over sufficiently small intervals of time. In contrast, because no interest is paid on intradaily positions and interest is instead paid discretely at the point when a position is rolled over from one day to the next, the size of the interest differential remains fixed over any interval that covers the time of the discrete interest payment. This is true no matter how short that interval is. Using a large dataset of high frequency exchange rate data, we run uncovered interest parity regressions over different time intervals. We replicate the rejection of uncovered interest parity with daily data, but find results that are consistently much more supportive of the uncovered interest parity hypothesis over short windows of intradaily data that span the time of the discrete interest payment.
\end{abstract}

Keywords: uncovered interest parity, high frequency data, exchange rates, risk premia.

JEL Classifications: C22, F31.

* Board of Governors of the Federal Reserve System. We are grateful to Kathryn Dominguez, Jon Faust, Dale Henderson, Mike Leahy and John Rogers for helpful comments. The views in this paper are solely the responsibility of the authors and should not be interpreted as reflecting the views of the Board of Governors of the Federal Reserve System or of any person associated with the Federal Reserve System. 


\section{Introduction}

The empirical failure of the simple uncovered interest parity (UIP) relation has been a puzzle to economists working in international finance ever since the work of Fama (1984). The UIP relation postulates that the interest differential between two countries should equal the expected exchange rate change. As such, a regression of exchange rate returns on the interest differential should give an intercept of zero and a slope coefficient of unity. This hypothesis has however been consistently and decisively rejected in the data. A carry trade (in which the investor borrows in the currency with the low interest rate and invests in the currency with a high interest rate) is profitable on average. Most often, the estimated slope coefficient is negative, meaning that the currency with the higher interest rate tends to appreciate.

Many comprehensive surveys exist (e.g. Froot and Thaler (1990) or Lewis (1995)) that list and discuss the explanations which economists have devised for the empirical failure of UIP. We do not attempt to review these, other than to note that the explanations include peso problems, learning effects, and the existence of a risk-premium (defined as the ex-ante expected profit on the carry trade) that is time-varying and correlated with the interest differential. Economists have not however had much success in explicitly modeling this risk premium, although attempts have been made to relate it to the relative cumulative current account balances of the two countries, or to the relative uncertainty in monetary policy in the two countries, among other things. 
Meredith and Chinn (1998) and Fujii and Chinn (2001) have considered running the UIP regression over long horizons. Exchange rate returns from $t$ to $t+m$ are regressed on the difference in the yields on $m$-period government bonds at time $t$. These authors have found that as the horizon $m$ increases, the rejection of the UIP hypothesis becomes less decisive. They interpret this as meaning that any risk premium is relatively stable over very long horizons.

In this paper, we are going in exactly the opposite direction, examining UIP over extremely short horizons. We will exploit the feature of money markets that interest is only paid on overnight positions. No interest is paid on intraday positions. Lyons and Rose (1995) is the only extant paper that has exploited this fact in looking at the relationship between interest differentials and exchange rates at high frequency, to the best of our knowledge. Lyons and Rose considered pairs of currencies in the now-defunct European Monetary System (EMS). They found that currencies which were under attack ${ }^{1}$ actually appreciated intraday, if the currency was not in fact devalued. Their interpretation of this finding is that investors must be compensated for the risk of devaluation. Overnight, they can be compensated by an interest differential. Intraday, there are however no interest differentials. So, if the currency stays within the band, it must appreciate in order to compensate them for the risk of devaluation that might have occurred, but did not.

\footnotetext{
${ }^{1}$ Concretely, Lyons and Rose (1995) considered the French franc-mark and lira-mark bilateral exchange rates, and defined the franc or lira as being under attack on those days on which the interest differential was in the top decile.
} 
We focus instead on the flip-side of the argument of Lyons and Rose (1995). Instead of looking at high frequency exchange rate movements over the intraday period when no interest is paid, we instead consider the overnight period when interest does accrue. Interest is paid on positions that are open at a particular point in time (17:00 New York time). If trading is liquid around this time, we should expect to see a jump in the exchange rate to offset the interest differential at this instant. Otherwise, an arbitrage opportunity exists as the investor can gain the interest differential while being exposed to exchange rate risk for an arbitrarily short period of time. This is entirely analogous to the discrete jump in a stock price when it goes ex-dividend. We therefore argue that an intraday UIP regression over a short period that spans 17:00 New York time may have the full interest differential, but a negligible risk premium. We test empirically whether or not this is the case, and find remarkably consistent affirmative results, using 10 years of high frequency exchange rate data on dollar bilateral exchange rates relative to the yen, mark, Swiss franc, and pound. One contribution of our paper relative to that of Lyons and Rose is that we run conventional UIP regressions (regressions of the exchange rate return on the interest differential) over windows of time that bracket the discrete payment of interest, instead of looking at the intraday period when no interest is paid. Also, our results are not focussed only on EMS currencies under speculative attack.

The plan for the remainder of this paper is as follows. Section 2 lays out the implications of the discrete timing of interest payments for high frequency UIP regressions. Section 3 
contains the empirical work. Section 4 concludes.

\section{Implications of the Discrete Timing of Interest Payments}

Let $s(t, h)$ denote the log exchange rate (foreign currency per dollar) on day $t$ at time $h$. Intraday interest rates are zero - only positions that are open overnight attract interest. A position that is open at a certain cutoff time is deemed to be held overnight and so attracts interest. This cutoff time is 17:00 New York time ${ }^{2}$. We adopt the convention that this time is the end of day $t$ and the start of day $t+1$. Let $i_{t}$ denote the dollar overnight interest rate from day $t$ to day $t+1$ and let $i_{t}^{*}$ denote the foreign overnight interest rate.

Consider the self-financing strategy of going short the foreign currency on day $t$ at time $h_{1}$, investing the proceeds in the domestic currency, and unwinding the position the next day at time $h_{2}$. Settlement in the foreign exchange market is $t+2^{3}$, which means that this investor will receive the interest differential prevailing between days $t+2$ and $t+3$ which is $i_{t+2}-i_{t+2}^{*}$. This is not exactly the same as the interest differential when the carry trade is initiated $\left(i_{t}-i_{t}^{*}\right)$, and is not even known at that time. This is a general issue in any uncovered interest parity regression, which we for now follow most of the literature in

\footnotetext{
${ }^{2}$ The 17:00 cutoff time (21:00 GMT during daylight savings time, 22:00 GMT at other times) is a rigid convention for EBS, the major electronic brokerage system. Any EBS quote flags whether the quote is before or after the cutoff time. Liquidity is however very thin around this time - positions that are open at this time in practice usually remain open at least until trading gets going in Tokyo.

${ }^{3}$ Parties to a trade are in principle free to fix settlement at any time they both agree to, but the two business day settlement lag is a very strong convention, for the currencies that we work with in the empirical part of this paper. Actually, settlement is $t+1$ for the Canadian dollar, but we do not consider the Canadian dollar in our empirical work. Stigum (1990) contains a comprehensive discussion of settlement issues.
} 
ignoring as small order, but shall discuss further in section 3 below. So we treat the return from the transaction of going short the foreign currency on day $t$ at time $h_{1}$, investing the proceeds in the domestic currency, and unwinding the position the next day at time $h_{2}$ as being

$$
s\left(t+1, h_{2}\right)-s\left(t, h_{1}\right)-\left(i_{t}^{*}-i_{t}\right)
$$

Define the expected return on this transaction ex-ante (i.e. on day $t$ at time $h_{1}$ ) as the risk-premium $R P\left(t, h_{1} ; t+1, h_{2}\right)$. By definition, in the equation

$$
s\left(t+1, h_{2}\right)-s\left(t, h_{1}\right)-\left(i_{t}^{*}-i_{t}\right)=R P\left(t, h_{1} ; t+1, h_{2}\right)+u_{t}
$$

the error term must be orthogonal to anything in the information set on day $t$ at time $h_{1}$, including the interest differential. Thus, in the equation

$$
s\left(t+1, h_{2}\right)-s\left(t, h_{1}\right)=\alpha+\beta\left(i_{t}^{*}-i_{t}\right)+R P\left(t, h_{1} ; t+1, h_{2}\right)+u_{t}
$$

the intercept coefficient $\alpha$ is zero, and the slope coefficient $\beta$ is one. As it stands, this equation is nothing more than an accounting identity. The UIP hypothesis however sets the risk premium to zero, requiring that the ex-ante expected return on the carry trade should always be zero, and so implies that in the regression

$$
s\left(t+1, h_{2}\right)-s\left(t, h_{1}\right)=\alpha+\beta\left(i_{t}^{*}-i_{t}\right)+u_{t}
$$

the intercept and slope coefficients should be zero and one, respectively. If we observe daily but not intradaily data - that is we observe the exchange rate at only a fixed time $h$ each 
day, then the regression equation simplifies further to

$$
s(t+1, h)-s(t, h)=\alpha+\beta\left(i_{t}^{*}-i_{t}\right)+u_{t}
$$

which is just the standard UIP relation, for daily data ${ }^{4}$.

The hypothesis that the slope coefficient is equal to one has been tested, and decisively rejected, for many currency pairs and sample periods. The standard interpretation of this result is that some risk premium exists which is time varying, and correlated with the interest differential. In this paper, we make no attempt to explicitly model the risk premium. We do however consider how to exploit high frequency intradaily data. Let $\lambda$ denote the time elapsed between time $h_{1}$ on day $t$ and time $h_{2}$ on day $t+1$. Our central assumption about the risk premium is that it is small over short intervals of time, specifically

$$
\lim _{\lambda \rightarrow 0} R P\left(t, h_{1} ; t+1, h_{2}\right)=0
$$

Crucially, however, no matter how little time elapses between time $h_{1}$ on day $t$ and time $h_{2}$ on day $t+1$, the carry trade still involves a fixed interest differential. The combination of the accounting identity in equation (3) and the assumption in equation (6) imply that equation (4) must hold with $\alpha=0$ and $\beta=1$, in a sufficiently small window around the time of the discrete interest payment. That is, $\alpha=0$ and $\beta=1$ if time $h_{1}$ is sufficiently late on day $t$, and time $h_{2}$ is sufficiently early the next day ${ }^{5}$.

\footnotetext{
${ }^{4}$ Here and throughout this paper, and in common with nearly all the UIP literature, we are neglecting a Jensen's inequality effect. This effect is numerically small, and is absorbed in the constant term, leaving the slope coefficient that is the primary object of interest unaffected.

${ }^{5}$ If we picked any arbitrarily short interval of time not spanning the time of the payment of the interest
} 
Neglecting transactions costs, under the condition in equation (6), it must be true that the slope and intercept coefficients in equation (4) are 0 and 1 , respectively, if time $h_{1}$ is sufficiently late on day $t$, and time $h_{2}$ is sufficiently early on day $t+1$. Otherwise an arbitrage opportunity exists. In this sense, testing this hypothesis is somewhat akin to a test of covered interest parity. However, the one-day interest differential is small - it is often of the order of 1 basis point. The simple strategy of shorting the low-interest currency at the very end of day $t$ and then unwinding the position at the start of day $t+1$ purely so as to pick up the interest differential is unlikely to be profitable, because of transactions $\operatorname{costs}^{6}$. Still, the investor deciding whether to conduct a transaction at the very end of day $t$, or at the start of day $t+1$, should care about the interest differential, and should take this into account in his or her decision. The presence of transactions costs might well prevent us from accepting the UIP hypothesis in a short window around the time of the interest payment, but need not necessarily do so. It is to find out that we will turn to empirical analysis in the next section.

differential, we might also expect the risk premium to shrink to zero, meaning that expected exchange rate change over that period of time would shrink to zero. But over an arbitrarily short interval of time that does span the time of the payment of the interest differential, if the risk premium shrinks to zero, the expected exchange rate change is not zero, but rather a jump to offset the interest differential.

${ }^{6}$ In the interdealer market for major currency pairs, the bid-ask spread is often of the order of 2 basis points, in times of active trading (e.g. Lyons (1995)). 


\section{Empirical Work}

Our spot exchange rate data consist of the exchange value of the Japanese Yen, German Mark, Swiss Franc and Pound Sterling (relative to the US Dollar) provided by Olsen and Associates every 5 minutes, covering the entire calendar years 1988 to 1998, inclusive. To construct these data, Olsen and Associates record all Reuters quotes, average the bid and the ask, and then linearly interpolate the resulting series to get prices at exactly the required times $^{7}$. We discard weekends, defined to be the time from 23:00 GMT on Friday to 22:55 GMT on Sunday, because there is virtually no foreign exchange trading during this time.

Our interest rate data consist of the overnight interbank interest rates for Japan, Germany, Switzerland and the United Kingdom, obtained from the BIS database, and the effective Federal Funds rate for the United States ${ }^{8}$. In our regressions we divide this interest rate by 360 . On Fridays, we then multiply this interest rate by 3 .

We first ran the ordinary UIP regression with daily data, equation (5), regressing the exchange rate returns from day $t$ to day $t+1$ on the overnight interest rate differential for each currency pair. The exchange rate we took for each day is as of 16:30 New York time. Here and throughout this paper we use heteroskedasticity-robust White standard errors ${ }^{9}$. The

\footnotetext{
${ }^{7}$ These are based on Reuters indicative quotes, not transaction prices. Danielsson and Payne (2002) compare Reuters indicative quotes and transactions prices, and find that the 5-minute returns on the two series are very highly correlated.

${ }^{8}$ We have also redone our empirical analysis using one-month interest rates from the 11am British Bankers Association (BBA) fixing, and get very similar results. These one-month interest rates are however not exactly the interest rates that would be used for overnight funding. The BBA now collects overnight interest rates in its daily fixing, but did not do so during our sample period.

${ }^{9}$ The standard errors are not autocorrelation-robust, because the holding periods for the carry trade are
} 
results are reported in Table 1. For all currencies except the pound, the estimated slope coefficient is negative, and is significantly different from one. Our span of data covers 10 years, shorter than in many UIP studies, leading to quite large standard errors. Nevertheless, our results are consistent with the general rejection of the UIP hypothesis. Over the 1990s, the rejection of UIP has been found by some authors to be less dramatic than in earlier periods (see, for example, Flood and Rose (2002)).

We next turned to running the proposed regression, as in equation (4), over a window from time $h_{1}$ to time $h_{2}$. In theory, as discussed in section 2 , we would like to select these times so as to construct the smallest possible window around 17:00 New York time. In practice, however we also want the markets to be liquid at these times. Also, we want to select the times $h_{1}$ and $h_{2}$ so that the data we use (based on linearly interpolated Reuters quotes) can be thought of as referring to prices before and after the rollover time as unambiguously as possible. We therefore set $h_{1}$ to 16:30 New York time (late afternoon trading in New York), and $h_{2}$ to 21:00 New York time (morning trading in Tokyo ${ }^{10}$ ). The results, reported in Table 2, are very different from those in the ordinary UIP regression. In all cases except the yen, the slope coefficient is estimated to be positive and is not significantly different from one. Even for the yen, the estimated coefficient is much higher than in the ordinary UIP regression with daily data. This is quite consistent with the idea of the risk premium being

non-overlapping.

${ }^{10}$ Tokyo time is 13 hours ahead of New York in summer, and 14 hours in winter. So, 21:00 New York time is 10:00 Tokyo time in summer, or 11:00 Tokyo time in winter (Japan does not have daylight savings time). 
small in short windows around the time of the discrete interest payment, as discussed in the previous section ${ }^{11}$.

Importantly, the regression is also much more precisely estimated over the period from 16:30 to 21:00. The reason why is simple. Over this period, the variance in the regressor (the interest differential) is the same as in the daily regression. The variance in the error term is however much lower. The signal-to-noise ratio is thereby more favorable to precise inference using the judiciously chosen intradaily interval that spans the actual interest payment. The error term $u_{t}$, like the risk premium, is of small order over this short period of time, but the interest differential is just the same as in an entire 24 hour period.

The slope coefficients in equation (4) setting $h_{1}$ to 16:30 New York time, and using various values of $h_{2}$ from 19:00 to 16:30 the next day (New York time) are plotted in Figure 1. A distinctive pattern can be seen in these plots, whereby the later $h_{2}$ is in the day, the lower the coefficient estimate is. The relationship is more or less monotone. Setting $h_{2}$ to 16:30 just gives a standard daily-frequency UIP regression. The pattern is especially dramatic for the mark and the Swiss franc, but can also be observed for the pound and the yen. This figure shows the central finding in the paper graphically - on average, currencies do indeed move in the direction predicted by UIP in short windows around the time of the discrete interest payment, but then move back the other way, and the latter effect dominates at the

\footnotetext{
${ }^{11}$ Meanwhile, it does not seem to us that this can easily be reconciled with the idea that the failure of UIP when not using intradaily data could be purely due to difficulties in statistical inference (as discussed by Baillie and Bollerslev (2000), among others), or due to expectational errors.
} 
daily frequency.

\subsection{Joint Estimation}

We can potentially exploit the fact of having four currencies to achieve more precise inference, estimating equation (5) on daily data, and equation (4) over the window from 16:30 to 21:00 for all currency pairs jointly, imposing that the slope coefficient is equal for all pairs, by the seemingly unrelated regression estimator. The slope coefficient estimated on daily data is -0.51 with a standard error of 0.48 . The slope coefficient estimated over the window from 16:30 to $21: 00$ is 0.60 , with a standard error of 0.17 . Although significantly below one, this is still much more supportive of the UIP hypothesis than the estimate obtained from daily data.

\subsection{Taking the Settlement Lag Seriously}

Settlement in the foreign exchange market is $t+2$. This means that, strictly, the interest differential that is actually received by the investor who conducts the carry trade that we describe (going short the foreign currency on day $t$ at time $h_{1}$, investing the proceeds in the domestic currency, and unwinding the position the next day at time $\left.h_{2}\right)$ is $i_{t+2}^{*}-i_{t+2}$. This is not even in the information set at the time that the carry trade is initiated. We can however adapt the UIP framework to take account of the settlement lag. It is perhaps more important to do this when working with daily and intradaily data, as in this paper, than in lower frequency UIP regressions. The vast majority of the UIP literature ignores the settlement lag issue. Bekaert and Hodrick (1993) is an exception, though they found that 
taking careful account of the settlement lag made little difference to their results.

What we would really like is an interest rate differential known on day $t$ that applies between the value date for a transaction on day $t$ and the value date for a transaction on day $t+1$ - that is an interest differential known on day $t$ that applies between days $t+2$ and $t+3$. By covered interest parity, this is the same as the difference between the spot rate on day $t$ (with value date $t+2$ ) and a forward rate for delivery the next day. Unfortunately, we do not have data on forward rates for delivery one day after the spot value date for our sample period. So we have to think of a slightly different adaptation of the UIP framework to take account of the settlement $\operatorname{lag}^{12}$.

The return from the transaction of going short the foreign currency on day $t$ at time $h_{1}$, investing the proceeds in the domestic currency, and unwinding the position the next day at time $h_{2}$ is strictly $s\left(t+1, h_{2}\right)-s\left(t, h_{1}\right)-\left(i_{t+2}^{*}-i_{t+2}\right)$, not the approximation given by equation (1) (see e.g. Loopesko (1984)). Rational expectations combined with risk neutrality imply that this trade should have a return that is orthogonal to anything in the information set at the time that the trade is initiated, including the interest differential observed at this time, $i_{t}^{*}-i_{t}$. So, in the regression

$$
s\left(t+1, h_{2}\right)-s\left(t, h_{1}\right)+\left[\left(i_{t}^{*}-i_{t}\right)-\left(i_{t+2}^{*}-i_{t+2}\right)\right]=\mu+\gamma\left(i_{t}^{*}-i_{t}\right)+v_{t}
$$

\footnotetext{
${ }^{12}$ Our approach to taking account of the settlement lag issue is therefore not exactly the same as that of Bekaert and Hodrick (1993). They were looking at UIP over a 30 day horizon, and had data on forward rates for delivery 30 days after the spot value date. Forward contracts for delivery 30 days after spot are standard, those for delivery 1 day after spot are not.
} 
the intercept and slope coefficients should be zero and one, respectively. This is the counterpart of equation (4) taking the settlement lag seriously, but can equivalently be thought of as a standard test for the predictability of the return on the carry trade, $s\left(t+1, h_{2}\right)-$ $s\left(t, h_{1}\right)-\left(i_{t+2}^{*}-i_{t+2}\right)$, given $i_{t}^{*}-i_{t}$. If we observe daily but not intradaily data - that is we observe the exchange rate at only a fixed time $h$ each day, then this simplifies to

$$
s(t+1, h)-s(t, h)+\left[\left(i_{t}^{*}-i_{t}\right)-\left(i_{t+2}^{*}-i_{t+2}\right)\right]=\mu+\gamma\left(i_{t}^{*}-i_{t}\right)+v_{t}
$$

which is a standard UIP regression for daily data, except with an adjustment to take account of the settlement lag.

The results from estimating the regression in equation (7), with $h_{1}$ being 16:30 New York time, and $h_{2}$ being set to 21:00 New York time are shown in Table 3. The results are even more favorable to the UIP hypothesis than in the counterpart of this equation that did not take account of the settlement lag. For all four currency pairs, the slope coefficient is estimated to be positive and not significantly different from one.

It is perhaps of some separate interest that taking the settlement lag seriously does seem to give results that are a little more favorable towards the UIP hypothesis. We also ran the UIP regression with daily data, but taking account of the settlement timing issue - i.e. estimating equation (8) taking the exchange rate for each day as of 16:30 New York time. The results are shown in Table 4. Just as in the ordinary UIP regression with daily data, for all currencies except the pound, the estimated slope coefficient is negative and significantly different from one. But the estimates are uniformly slightly higher than in 
estimating equation (5).

\subsection{Robustness Checks}

As a simple robustness check, to guard against results being driven by outliers, we calculated the proportion of times that the exchange rate change had the correct sign as predicted by UIP, both at the daily frequency, and in the period from 16:30 to 21:00. Where the foreign interest rate is greater than the US interest rate, UIP would call for the dollar to appreciate, and vice-versa. The fraction of days on which this prediction is in fact correct is shown in Table 5, along with standard errors. For all currencies except the pound, using data at the daily frequency, the estimated proportion of times that UIP predicts the correct sign is less than half. So, at the daily frequency, UIP is wrong more often than it is right. However, over the intradaily window from 16:30 to 21:00, UIP does better than at the daily frequency for all four currencies. In this intradaily window, UIP makes the correct prediction about the direction of the exchange rate movement more than half the time for the pound, mark, and Swiss franc. The direction of the exchange rate change is in line with UIP $56 \%$ of the time for the pound. This is significantly better than a coin toss, although the exchange rate movement does still often go the wrong way, which just confirms that exchange rates are very noisy. We are able to do reasonably precise inference only because we have a sample spanning 10 years.

As a final robustness check, we re-ran the baseline regressions in Tables 1 and 2 but using only days for which the interest differential exceeded 4 percentage points in absolute 
magnitude. The slope coefficients are reported in Table 6. The standard errors are larger, but the results over the period from 16:30 to 21:00 are dramatically more supportive of UIP than are the results at the daily frequency. Table 7 shows the proportion of days on which the interest differential exceeded 4 percentage points in absolute magnitude that the exchange rate change had the correct sign as predicted by UIP, both at the daily frequency, and in the period from 16:30 to 21:00. For all currencies, using data at the daily frequency, when the interest differential is large, UIP is a worse predictor than a coin toss. But except for the yen, this is reversed in the intradaily window from 16:30 to 21:00. For sterling, the fraction of days that UIP makes the right sign prediction rises from $48 \%$ in the daily data to $57 \%$.

\section{Conclusion}

No interest is paid on intradaily positions. Rather, interest is paid discretely, at the point when a position is rolled over from one day to the next. The common rollover time is determined by market convention. This practice has potential implications for high-frequency exchange rate movements.

Uncovered interest parity is both central to theoretical models, and an enormous empirical failure. This widely found empirical failure can be ascribed to the existence of a risk premium. The size of this risk premium may however shrink to zero over sufficiently small intervals of time. In contrast, because of the market practice of discrete interest payments, 
the size of the interest differential remains fixed over any interval that covers the time of the discrete interest payment, no matter how short the interval.

Using a large dataset of high frequency exchange rate data, covering mark, yen, pound and Swiss franc exchange rates viz-a-viz the US dollar, we run uncovered interest parity regressions over different time intervals. We replicate the rejection of uncovered interest parity with daily data, but find results that are uniformly substantially more supportive of the uncovered interest parity hypothesis over short windows of intradaily data that span the time of the discrete interest payment. Of course, it remains very much a puzzle that time variation in the risk premium at the daily frequency is sufficiently important as to lead to the rejection of uncovered interest parity at that frequency.

\section{References}

Baillie, R.T. and T. Bollerslev (2000): The Forward Premium Anomaly is Not as Bad as You Think, Journal of International Money and Finance, 19, pp.471-488.

Bekaert, G. and R.J. Hodrick (1993): On Biases in the Measurement of Foreign Exchange Risk Premiums, Journal of International Money and Finance, 12, pp.115-138.

Danielsson, J. and R. Payne (2002): Real Trading Patterns and Prices in Spot Foreign Exchange Markets, Journal of International Money and Finance, 21, pp.203-222.

Fama, E.F. (1984): Forward and Spot Exchange Rates, Journal of Monetary Economics, 14, pp.319-338. 
Flood, R.P. and A.K. Rose (2002): Uncovered Interest Parity in Crisis, IMF Staff Papers, 49, pp.252-266.

Froot, K.A. and R.H. Thaler (1990): Anomalies: Foreign Exchange, Journal of Economic Perspectives, 4, pp.179-192.

Fujii, E. and M. Chinn (2001): Fin de Siecle Real Interest Parity, Journal of International Financial Markets, Institutions and Money, 11, pp.289-308.

Lewis, K.K. (1995): Puzzles in International Financial Markets in "Handbook of International Economics", Volume 3, G.M. Grossman and K. Rogoff eds., Elsevier, New York.

Loopeseko, B.E. (1984): Relationships Among Exchange Rates, Intervention, and Interest Rates, Journal of International Money and Finance, 3, pp.257-277.

Lyons, R.K. (1995): Tests of Microstructural Hypotheses in the Foreign Exchange Market, Journal of Financial Economics, 39, pp.321-351.

Lyons, R.K. and A.K. Rose (1995): Explaining Forward Exchange Bias ... Intraday, Journal of Finance, 50, pp.1321-1329.

Meredith, G. and M. Chinn (1998): Long-Horizon Uncovered Interest Rate Parity, National Bureau of Economic Research Working Paper 6797.

Stigum, M. (1990): The Money Market, McGraw Hill, New York. 
Table 1: Standard UIP Regressions for Daily Data on U.S.-foreign bilateral exchange rate Coefficient Estimates (Standard Errors in Parentheses)

\begin{tabular}{rrr}
\hline \hline Currency & Intercept & Slope \\
& & \\
Swiss Franc & -0.01 & -2.03 \\
& $(0.02)$ & $(1.02)$ \\
& & \\
Mark & 0.00 & -1.36 \\
& $(0.01)$ & $(1.03)$ \\
& & \\
Pound & 0.00 & 0.11 \\
& $(0.01)$ & $(1.13)$ \\
& & \\
Yen & -0.02 & -1.75 \\
& $(0.02)$ & $(0.97)$ \\
\hline \hline
\end{tabular}

Notes: This table reports the results of the UIP regression with daily data, measured at 16:30 New York time each day, i.e. the regression in equation (5) with $h$ set to 16:30 New York Time. White standard errors are reported.

Table 2: UIP Regressions from 16:30 to 21:00 New York Time Coefficient Estimates (Standard Errors in Parentheses)

\begin{tabular}{rrr}
\hline \hline Currency & Intercept & Slope \\
& & \\
Swiss Franc & -0.01 & 0.59 \\
& $(0.01)$ & $(0.45)$ \\
& & \\
Mark & 0.00 & 0.54 \\
& $(0.00)$ & $(0.51)$ \\
& & \\
Pound & 0.01 & 0.87 \\
& $(0.00)$ & $(0.50)$ \\
& & \\
Yen & 0.00 & -0.31 \\
& $(0.01)$ & $(0.50)$ \\
\hline \hline
\end{tabular}

Notes: This table reports the results of the UIP regression with intradaily data, from 16:30 to 21:00 New York time each day, i.e. the regression in equation (4) with $h_{1}$ and $h_{2}$ set to 16:30 and 21:00 New York Time, respectively. White standard errors are reported. 
Table 3: UIP Regressions from 16:30 to 21:00 With Adjustment for the Settlement Lag Coefficient Estimates (Standard Errors in Parentheses)

\begin{tabular}{rrr}
\hline \hline Currency & Intercept & Slope \\
& -0.00 & 0.95 \\
Swiss Franc & $(0.01)$ & $(0.45)$ \\
& & \\
Mark & -0.00 & 0.87 \\
& $(0.00)$ & $(0.51)$ \\
& & \\
Pound & 0.01 & 1.44 \\
& $(0.00)$ & $(0.51)$ \\
& 0.00 & 0.17 \\
Yen & $(0.01)$ & $(0.50)$ \\
\hline \hline
\end{tabular}

Notes: This table reports the results of the UIP regression with intradaily data, from 16:30 to 21:00 New York time each day but taking account of the two-day settlement lag, i.e. the regression in equation (7) with $h_{1}$ and $h_{2}$ set to 16:30 and 21:00 New York Time, respectively. White standard errors are reported.

Table 4: UIP Regressions for Daily Data With Adjustment for the Settlement Lag Coefficient Estimates (Standard Errors in Parentheses)

\begin{tabular}{rrr}
\hline \hline Currency & Intercept & Slope \\
& & \\
Swiss Franc & -0.01 & -1.68 \\
& $(0.01)$ & $(1.02)$ \\
Mark & -0.01 & -1.05 \\
& $(0.01)$ & $(1.03)$ \\
& & \\
Pound & 0.00 & 0.69 \\
& $(0.02)$ & $(1.13)$ \\
& & \\
Yen & -0.02 & -1.28 \\
& $(0.02)$ & $(0.97)$ \\
\hline \hline
\end{tabular}

Notes: This table reports the results of the UIP regression with daily data, measured at 16:30 New York time each day, but taking account of the two-day settlement lag, i.e. the regression in equation (8) with $h$ set to 16:30 New York Time. White standard errors are reported. 
Table 5: Percentage of Days when the UIP Predicts the Correct Sign of the Exchange Rate Change

\begin{tabular}{rrr}
\hline \hline Currency & 16:30-16:30 Change & 16:30-21:00 Change \\
Swiss Franc & 49.4 & 52.3 \\
Mark & 48.5 & 52.3 \\
Pound & 50.2 & 56.3 \\
Yen & 48.4 & 49.7 \\
\hline \hline
\end{tabular}

Notes: This table reports the fraction of days in which the currency with the lower interest rate appreciates, as predicted by UIP, both in the period from 16:30 to 16:30 the next day, and in the period from 16:30 to 21:00, New York Time. The standard error for each of these as estimates of the true probability that UIP predicts the correct sign is 0.9 percentage points, using the standard formula for the variance of a binomial distribution.

Table 6: Slope Coefficients in UIP Regression Over Different Windows Version deleting days when the absolute interest differential is no greater than $4 \%$

\begin{tabular}{|c|c|c|}
\hline Currency & "16:30-16:30 Change & 16:30-21:00 Change \\
\hline Swiss Franc & $\begin{array}{r}-2.87 \\
(1.57)\end{array}$ & $\begin{array}{r}1.06 \\
(0.79)\end{array}$ \\
\hline Mark & $\begin{array}{r}-3.60 \\
(1.84)\end{array}$ & $\begin{array}{r}0.23 \\
(0.99)\end{array}$ \\
\hline Pound & $\begin{array}{r}-0.64 \\
(1.98)\end{array}$ & $\begin{array}{r}0.71 \\
(0.84)\end{array}$ \\
\hline Yen & $\begin{array}{r}-1.87 \\
(1.83)\end{array}$ & $\begin{array}{r}-0.99 \\
(0.97)\end{array}$ \\
\hline
\end{tabular}

Notes: This table reports the slope coefficients from the regressions in Tables 1 and 2, but deleting all days for which the interest differential is less than or equal to 4 percentage points. White standard errors are reported. 
Table 7: Percentage of Days when the UIP Predicts the Correct Sign of the Exchange Rate Change

Version deleting days when the absolute interest differential is no greater than $4 \%$

(Standard Errors in Parentheses)

\begin{tabular}{crr}
\hline \hline Currency & $16: 30-16: 30$ Change & $16: 30-21: 00$ Change \\
& & \\
Swiss Franc & 49.4 & 52.3 \\
& $(1.8)$ & $(1.8)$ \\
Mark & 48.5 & 52.3 \\
& $(2.2)$ & $(2.2)$ \\
Pound & 50.2 & 56.3 \\
& $(1.7)$ & $(1.6)$ \\
Yen & 48.4 & 49.7 \\
& $(1.4)$ & $(1.4)$ \\
\hline \hline
\end{tabular}

Notes: This table reports the fraction of days in which the currency with the lower interest rate appreciates, as predicted by UIP, both in the period from 16:30 to 16:30 the next day, and in the period from 16:30 to 21:00, New York Time, as in Table 5, , but deleting all days for which the interest differential is less than or equal to 4 percentage point. The standard errors for each of these as estimates of the true probability that UIP predicts the correct sign were obtained using the standard formula for the variance of a binomial distribution. Unlike in Table 5 they are different for different currency pairs, because the number of days with an absolute interest differential greater than 4 percentage points differs across country pairs. 
Fig. 1: Estimate of $\beta$ in Equation 4, with $90 \%$ Confidence Interval, Estimated from $16: 30$ to time $h_{2}$, plotted against $h_{2}$
\[ \text { Swiss Franc } \]

Swiss Franc

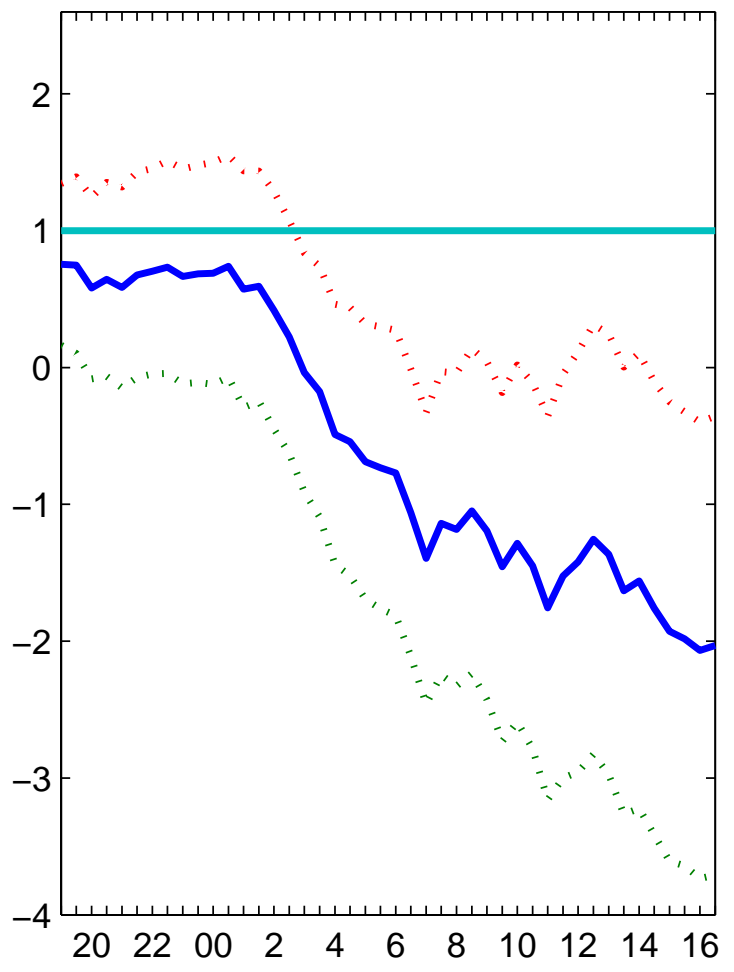

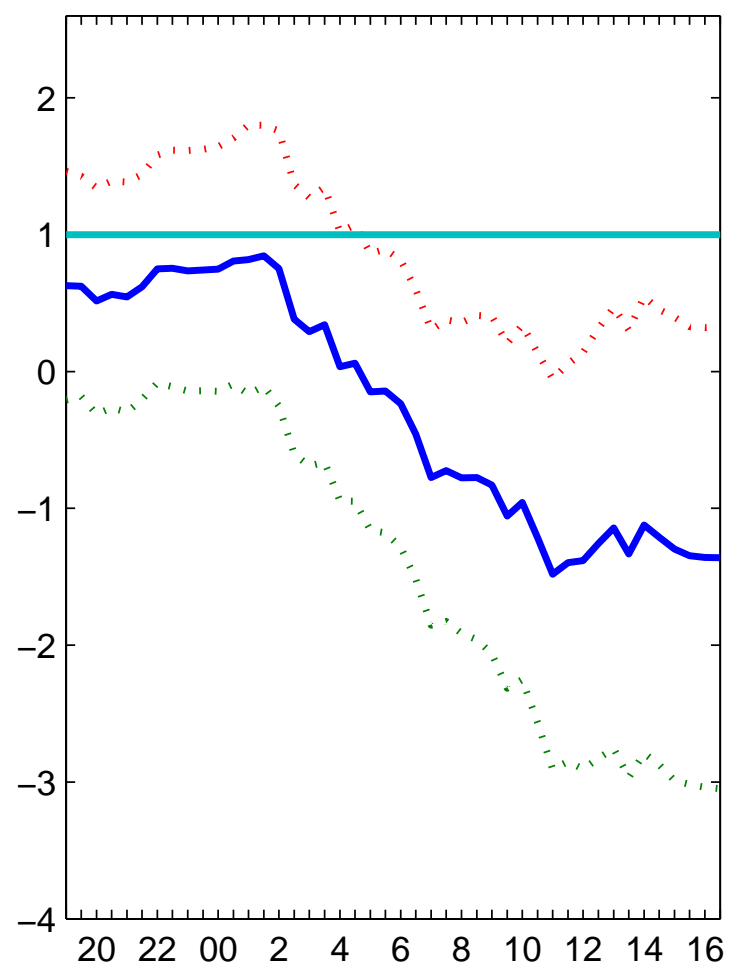

Pound

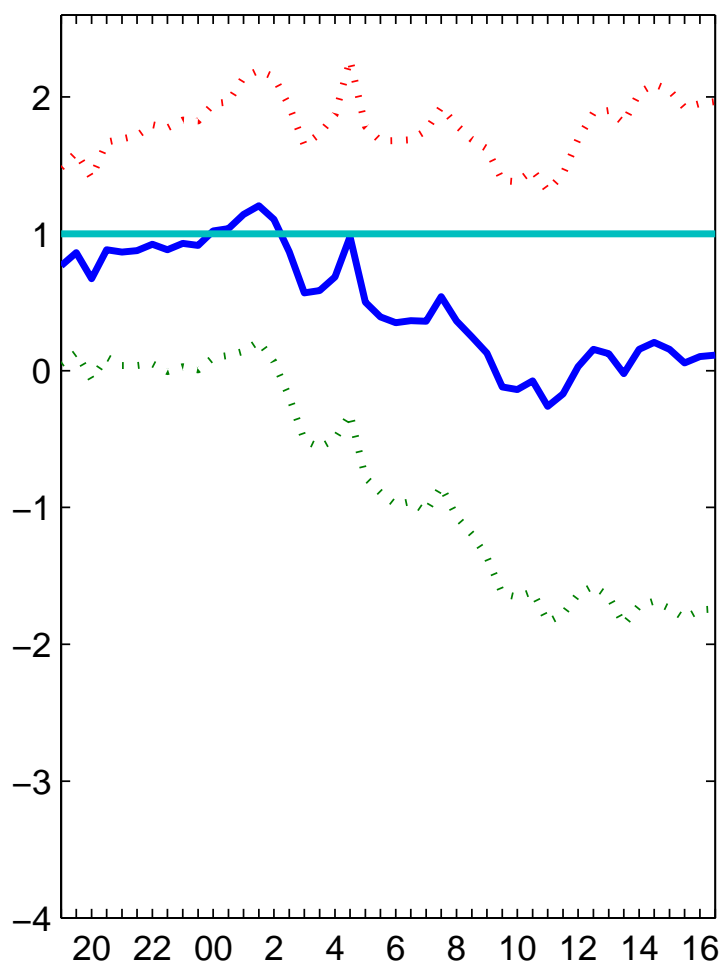
Notes: Time shown is New York Time

Yen

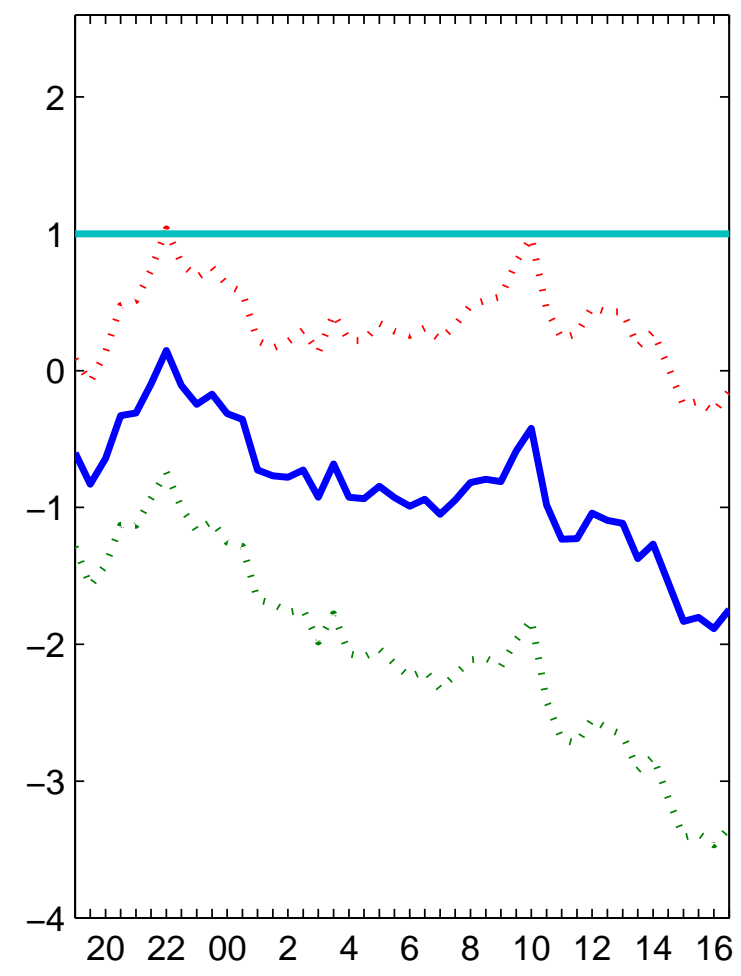

